\title{
Reporte de pérdidas en el rendimiento de la soja causadas por la mancha anillada (Corynespora cassiicola Berk. \& Curt.) en la Orinoquia colombiana
}

\section{Report of soybean yield losses caused by target spot (Corynespora cassiicola Berk. \& Curt) in the Colombian Orinoquia}

\author{
Deisy Lorena Flórez-Gómez; Samuel Caicedo-Guerrero²; Nathali López-Cardona ${ }^{3 *}$
}

${ }^{1}$ Ing. Agrónoma. Corporación Colombiana de Investigación Agropecuaria AGROSAVIA, Sede Central. Mosquera - Cundinamarca, Colombia; e-mail: dlflorez@agrosavia.co; iD https://orcid.org/0000-0003-3676-7564

${ }^{2}$ Ing. Agrónomo, M.Sc. Corporación Colombiana de Investigación Agropecuaria AGROSAVIA, Centro de Investigación La Libertad. Villavicencio - Meta, Colombia; e-mail: scaicedo@agrosavia.co; iD https://orcid.org/0000-0002-5822-5881

${ }^{3}$ Ing. Agrónoma, M.Sc. Corporación Colombiana de Investigación Agropecuaria AGROSAVIA, Centro de Investigación La Libertad. Villavicencio - Meta, Colombia; e-mail: nlopezc@agrosavia.co; iD https://orcid.org/0000-0001-8790-9070

*autor para correspondencia: nlopezc@agrosavia.co

Cómo citar: Flórez-Gómez, D.L.; Caicedo-Guerrero, S.; López-Cardona; N. 2021. Reporte de pérdidas en el rendimiento de la soja causadas por la mancha anillada (Corynespora cassiicola Berk. \& Curt.) en la Orinoquia colombiana. Rev. U.D.C.A Act. \& Div. Cient. 24(2):e1869. http://doi.org/10.31910/rudca.v24.n2.2021.1869

Artículo de acceso abierto publicado por Revista U.D.C.A Actualidad \& Divulgación Científica, bajo una Licencia Creative Commons CC BY-NC 4.0

Publicación oficial de la Universidad de Ciencias Aplicadas y Ambientales U.D.C.A, Institución de Educación Superior Acreditada de Alta Calidad por el Ministerio de Educación Nacional.

Recibido: febrero 17 de $2021 \quad$ Aceptado: agosto 2 de $2021 \quad$ Editado por: Ingeborg Zenner de Polanía

\section{INTRODUCCIÓN}

En el 2017, al realizar una Prueba de Evaluación Agronómica (PEA) de nuevas líneas de soja (Glycine max L.), para la Orinoquía Colombiana, Caicedo-Guerrero et al. (2020) reportaron la incidencia de la mancha anillada, en tres localidades de la Altillanura plana para los materiales evaluados. El patógeno fue determinado por López-Cardona et al. (2020) como el hongo Corynespora cassiicola, siendo el primer reporte de la enfermedad en soja, para la zona en el país. La mancha anillada es una enfermedad foliar común de la soja en los trópicos y subtrópicos (Dixon et al. 2009). Afecta todos los órganos de la planta, inclusive raíces, pero el síntoma más evidente son lesiones circulares, anillos concéntricos e irregulares, de color marrón-rojizo, rodeadas por un halo característico de color amarillo en las hojas. En cultivares altamente susceptibles puede causar defoliación prematura (Escobar et al. 2019; Sinclair, 1999). El primer reporte de C. cassiicola causando mancha anillada en la soja en Colombia, data de hace más de 2 décadas (BuriticáCéspedes, 1999); sin embargo, el patógeno ahora ha reemergido y representa una amenaza.

La mancha anillada, se ha extendido a todas las regiones productoras de soja en Brasil (Godoy et al. 2015) e, incluso, en Argentina, donde fue la enfermedad más prevalente, durante la temporada 2014-2015 (De Lisi et al. 2016). En el sureste de los Estados Unidos, el hongo resurgió entre el 2004-2005, probablemente, como consecuencia 
de los cambios en el patrón climático, la virulencia del patógeno o la introducción de genotipos de soja más susceptibles (Wrather \& Koenning, 2006). El objetivo de este estudio fue realizar un registro de pérdidas en el rendimiento, asociadas a la mancha anillada $(C$. cassiicola), en cuatro variedades de soja, establecidas en condiciones edafoclimáticas de la Altillanura plana, del departamento del Meta.

\section{MATERIALES Y MÉTODOS}

Durante marzo a agosto del 2018, se presentó una epidemia de mancha anillada en las variedades de soja: Corpoica Guayuriba 9, BRS 314 y BRS Corisco, Corpoica Achagua 8, establecidas en los lotes de mejoramiento de soja, en la Sede Taluma, del Centro de Investigación la Libertad de AGROSAVIA, ubicada en el kilómetro 91, vía a Puerto Gaitán (4²2'41', latitud Norte; $72^{\circ} 13^{\prime} 25^{\prime \prime}$, longitud Oeste; altitud de 175 m s.n.m.). La identidad del patógeno fue corroborada mediante la metodología de López-Cardona et al. (2020). Se registró el porcentaje de severidad de la enfermedad en condiciones de inóculo natural, a los 38,54, 72, 80, 88 y 94 días después de la emergencia (DDE), de acuerdo con la escala diagramática, propuesta por Soares et al. (2009) y, posteriormente, se estimó la tasa de infección de la enfermedad (r) y el Área Bajo La Curva de Progreso de la Enfermedad (ABCPE) (Castaño-Zapata, 2002). En un diseño de bloques completos al azar (DBCA), con cuatro tratamientos (cuatro variedades) y tres repeticiones y con parcelas de seis surcos (dos de efecto borde y cuatro fueron de parcela útil), de cinco metros de longitud y $0,50 \mathrm{~m}$ de distancia entre surcos, como unidad experimental. A partir de la evaluación de cinco plantas por parcela, se calcularon las siguientes variables: índice de carga, medido como la altura desde la superficie del suelo hasta la inserción de la primera vaina y expresada en centímetros; la severidad foliar de la mancha anillada, evaluada a los 82 días después de emergencia, con la escala diagramática de Soares et al. (2009); el número de vainas vanas, definida de acuerdo con el número de vainas sin semillas por planta y expresada en número; número de abortos embrionarios, calculada como el número de embriones no desarrollados en el total de vainas por planta y expresada en número; número de vainas totales, determinada por la cantidad de vainas por planta y expresada en número; peso de cien semillas, medida por el peso de cien semillas cosechadas por parcela y, el rendimiento de grano, calculado a partir del peso del grano por parcela, con $14 \%$ de humedad y expresado en toneladas por hectárea. Los datos obtenidos, se analizaron mediante el procedimiento de Análisis de Varianza -ANOVA-, del programa geoestadístico R Studio ${ }^{\circledR}$ y se realizó la prueba de comparación de medias de Tukey, con un nivel de significancia de 0,05.

\section{RESULTADOS Y DISCUSIÓN}

Los síntomas causados por el patógeno incluyeron necrosis en tallo, en peciolos y en manchas anilladas en hojas (Figura 1). Para las variedades menos afectadas: BRS Corisco $(1,4 \%$ de severidad promedio) y el testigo local Corpoica Achagua 8 (2,0\% de severidad promedio); la enfermedad presentó síntomas típicos solamente en las hojas del tercio inferior hacia el final del ciclo productivo, con una ligera defoliación prematura. Se cálculo de la tasa de infección (r) y el ABCPE, a partir de los registros de la severidad, a los 38, 54, 72, 80, 88 y 94 días después de la emergencia, permitió concluir que la enfermedad se desarrolla con mayor velocidad en la variedad BRS 314 ( $r=1,73 ;$ ABCPE = 514,37), seguida de las variedades Corpoica Guayuriba 9, el testigo local Corpoica Achagua 8 y BRS Corisco (Tabla 1). Se observó que el desarrollo de la enfermedad fue más lento en la variedad BRS Corisco $(\mathrm{r}=$ $1,12 ; \mathrm{ABCPE}=35,94)$, lo que sugiere tolerancia al patógeno, en comparación con las otras variedades evaluadas. La variedad más afectada por la mancha anillada fue BRS 314, cuyos registros de severidad promedio fueron del $52 \%$, situación que se refleja en el menor rendimiento obtenido de todas las variedades $(2,05 \mathrm{t} /$ ha). La variedad menos afectada fue BRS Corisco, con un registro de severidad promedio de $1,4 \%$, sin afectación de pérdidas en rendimiento. El testigo local Corpoica Achagua 8 y la variedad Corpoica Guayuriba 9, obtuvieron severidades promedio del 2 y $11,53 \%$, con rendimientos de 2,13 y $2,39 \mathrm{t} / \mathrm{ha}$, respectivamente.

De acuerdo con el análisis de los rendimientos comerciales y los componentes de rendimiento reportados por Caicedo et al. (2020) e ICA (2013a, 2013b) y los obtenidos en el ensayo (Tabla 1), la variedad BRS 314 disminuyó la producción de grano en $450 \mathrm{~kg} /$ ha, cuando presentó una severidad de la mancha anillada promedio de $52 \%$, lo que se traduce en una pérdida de rendimiento del

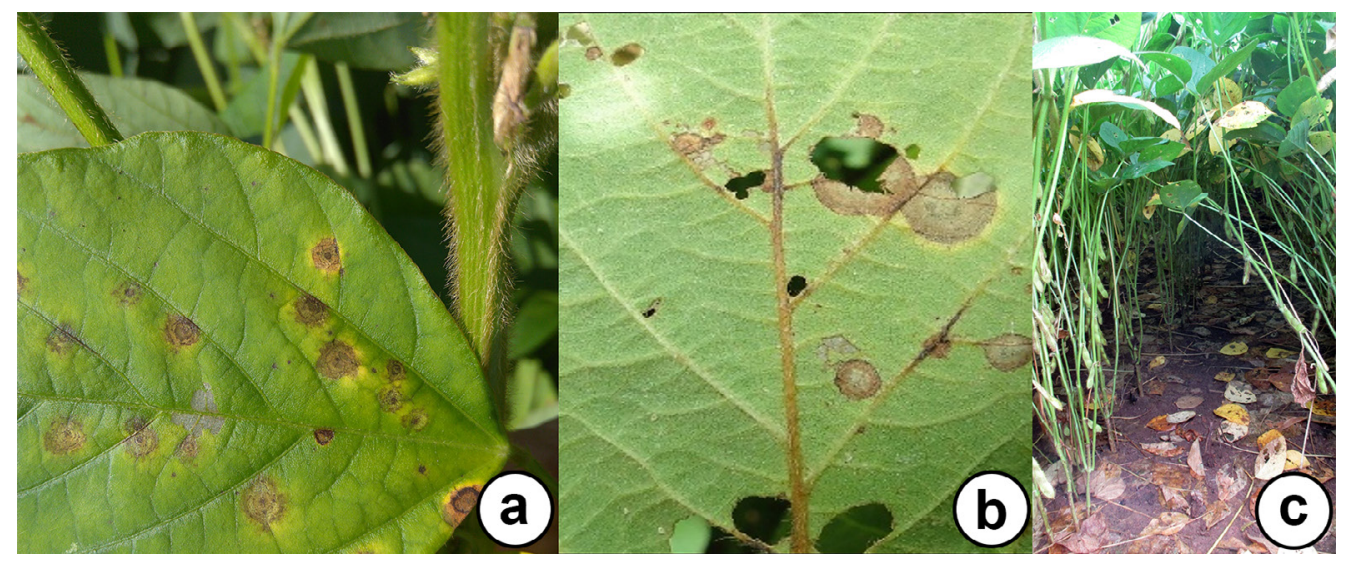

Figura 1. Síntomas de la mancha anillada (Corynespora cassiicola) en la variedad BRS 314. a. Síntoma típico de manchas anilladas; b. Necrosis de nervaduras; c. Defoliación. 
Tabla 1. Área Bajo la Curva del progreso de la mancha anillada (Corynespora cassiicola), tasa de infección (r) y variables teóricas asociadas al rendimiento, de cuatro variedades de soya, establecidas en la Sede Taluma, de AGROSAVIA.

\begin{tabular}{|c|c|c|c|c|}
\hline \multirow{2}{*}{ Variable } & \multicolumn{4}{|c|}{ Genotipos } \\
\hline & BRS 314 & BRS Corisco & C. Guayuriba 9 & C. Achagua $8^{2}$ \\
\hline $\mathrm{ABCPE}^{1}$ & 514,37 & 35,94 & 217,73 & 44,14 \\
\hline Tasa (r) de infección & 1,73 & 1,12 & 1,34 & 1,31 \\
\hline Rendimiento reportado (ton $/ \mathrm{ha})^{2}$ & 2,5 & 2,6 & 2,6 & 2,4 \\
\hline $\begin{array}{l}\text { Incremento }(+) \text { o decremento }(-) \text { en } \\
\text { rendimiento }(\mathrm{kg} / \mathrm{ha})(\%)\end{array}$ & $(-) 450(18)$ & $(+) 290(11)$ & $(-) 210(8)$ & $(-) 270(11)$ \\
\hline Índice de carga reportado $(\mathrm{cm})^{3}$ & 17,7 & 14,2 & 18 & 20 \\
\hline $\begin{array}{l}\text { Incremento }(+) \text { o decremento }(-) \text { en } \\
\text { índice de carga }(\mathrm{cm})(\%)\end{array}$ & $(+) 3,17(17,9)$ & $(+) 2,47(17,4)$ & $(+) 0,8(4,4)$ & 0 \\
\hline Hábito de crecimiento $^{4}$ & Indeterminado & Indeterminado & Determinado & Indeterminado \\
\hline
\end{tabular}

1/ Área Bajo la Curva del Progreso de la Enfermedad. 2,3/ Rendimiento comercial e índice de carga reportado por Caicedo et al. (2020) en cultivo de soja aparentemente sano en la altillanura plana del departamento del Meta. ${ }^{4}$ Caicedo et al. (2020).

$18 \%$; Corpoica Achagua 8 redujo la producción de grano en $270 \mathrm{~kg} / \mathrm{ha}$, cuando presentó una severidad promedio de $2 \%$, lo que se convierte en una pérdida de rendimiento del $11 \% \mathrm{y}$, por último, Corpoica Guayuriba 9, disminuyó la producción de grano en $210 \mathrm{~kg} / \mathrm{ha}$, cuando registró una severidad promedio de 11,53 $\%$ e impacto en pérdida de rendimiento del $8 \%$. Contrario a lo esperado con la variedad BRS Corisco, que presentó, durante todo el ciclo de cultivo, una alta tolerancia a la enfermedad, al presentar valores promedio de 1,4\% de severidad (Tabla 1).

Por otra parte, al analizar los componentes de rendimiento, se observó que todos los materiales, excepto el testigo local C. Achagua 8 , tuvieron un incremento en su índice de carga, lo cual, ratifica su adaptación específica a las condiciones de la Altillanura y su bajo grado de afectación por la enfermedad; no tuvo una alteración en la altura de inserción de la primera vaina, por el contrario, se observa que en las variedades BRS 314 y C. Guayuriba 9, la altura de carga fue mayor, lo que soporta una reducción (17,9 y 4,4 \%, respectivamente), en su capacidad de formar vainas a menor altura $\mathrm{y}$, por ende, se ve afectado el rendimiento de dichos materiales. En el caso de la variedad BRS Corisco, igualmente, se detalla un aumento en su índice de carga, en $2,47 \mathrm{~cm}$; sin embargo, el rendimiento no se afectó, dado a que, por su hábito de crecimiento indeterminado, pudo desarrollar nuevas estructuras vegetativas y reproductivas, logrando escapar a la enfermedad.

Por otro lado, los componentes de rendimiento más afectados por la enfermedad fueron el peso de 100 semillas y el número de vainas vanas y la cantidad de abortos embrionarios (Tabla 2). El índice de carga y el número de vainas totales por planta no presentaron diferencias estadísticamente significativas, entre las variedades evaluadas. Se resalta que la variedad BRS Corisco presentó el menor número de vainas vanas y de abortos embrionarios por planta, pero, a su vez, presentó el menor peso de 100 semillas. La

Tabla 2. Comparación de medias de la severidad de la mancha anillada y 6 variables asociadas al rendimiento, en cuatro variedades de soja, establecidas en la Sede Taluma, de AGROSAVIA.

\begin{tabular}{|c|c|c|c|c|c|c|}
\hline \multicolumn{7}{|c|}{ COMPARACIÓN DE MEDIAS ${ }^{1}$} \\
\hline \multirow{2}{*}{ Variable } & \multicolumn{4}{|c|}{ Genotipo } & \multirow{2}{*}{ Media } & \multirow{2}{*}{$\begin{array}{l}\text { C.V } \\
(\%)\end{array}$} \\
\hline & BRS 314 & $\begin{array}{c}\text { BRS } \\
\text { Corisco } \\
\end{array}$ & C. Guayuriba 9 & C. Achagua $8^{2}$ & & \\
\hline Severidad $(\%)$ & $52,00 \mathrm{a}$ & $1,40 \mathrm{c}$ & $11,53 \mathrm{~b}$ & $2,00 \mathrm{c}$ & 16,73 & 129,07 \\
\hline Rendimiento calculado (ton/ha) & $2,05 \mathrm{~d}$ & $2,89 \mathrm{a}$ & $2,39 \mathrm{~b}$ & $2,13 \mathrm{c}$ & 2,37 & 14,34 \\
\hline Índice de carga $(\mathrm{cm})$ & $20,87 \mathrm{a}$ & $16,67 \mathrm{a}$ & $18,80 \mathrm{a}$ & $20,00 \mathrm{a}$ & 19,08 & 30,73 \\
\hline Vainas vanas $(\mathrm{n})$ & $1,667 \mathrm{ab}$ & $0,533 \mathrm{~b}$ & $2,667 \mathrm{a}$ & $0,930 \mathrm{~b}$ & 1,45 & 132,22 \\
\hline Abortos embrionarios (n) & $13,40 \mathrm{ab}$ & $9,40 \mathrm{~b}$ & $18,60 \mathrm{a}$ & $16,53 \mathrm{ab}$ & 14,48 & 61,1 \\
\hline Vainas totales (n) & $89,26 \mathrm{a}$ & $68,40 \mathrm{a}$ & $95,66 \mathrm{a}$ & $63,60 \mathrm{a}$ & 79,23 & 47,44 \\
\hline Peso de 100 semillas $(\mathrm{g})$ & $16,47 \mathrm{c}$ & $14,22 \mathrm{~d}$ & $16,96 \mathrm{~b}$ & $19,29 \mathrm{a}$ & 16,74 & 11,24 \\
\hline
\end{tabular}

1/Medias con letras iguales en sentido vertical no son estadísticamente diferentes con $\mathrm{p} \leq 0,05$ (Tukey). ${ }^{2} /$ Testigo local. $^{1}$ 
variedad C. Guayuriba 9 registró el mayor número de vainas vanas y abortos embrionarios por planta. En cuanto al peso de 100 semillas, el testigo local C. Achagua 8, obtuvo un promedio de 19,3 gramos, a diferencia de la variedad BRS Corisco, cuya media para esta variable fue de 14,2 gramos, resultado que corrobora, nuevamente, la adaptación de la variedad a las condiciones edafoclimáticas de la Altillanura plana. Estos resultados son promisorios y demuestran que el cálculo de este componente de rendimiento es una variable muy importante, que contribuye al entendimiento de la tolerancia de la variedad C. Achagua 8 al ataque de C. cassiicola.

Los efectos de la mancha anillada en el rendimiento de la soja han sido reportados en varios estudios con resultados inconsistentes, con informes de ninguna pérdida (Ploper et al. 2013), hasta una reducción del rendimiento del $40 \%$, en otros casos (Sakamoto \& Shaw, 1967; Koenning et al. 2006; Edwards Molina et al. 2019). Estos resultados concuerdan con los obtenidos en el presente estudio y corroboran los reportes de Koenning et al. (2006) y Edwards Molina et al. (2019), quienes informan que las diferencias en la afectación del rendimiento son, probablemente, debidas a diferencias en la tolerancia entre las variedades. Los hallazgos obtenidos en el presente estudio demuestran que, en la región de la Altillanura plana colombiana, la mancha anillada, con una severidad entre el 2 al $52 \%$, puede causar pérdidas en el rendimiento entre el 8 al $18 \%$, dependiendo de la susceptibilidad de la variedad de soja, convirtiéndose en una enfermedad de importancia económica para el cultivo en Colombia; sin embargo, el ensayo deberá ser replicado en otro semestre productivo de soja, para que los resultados sean validados en la región.

Conflicto de intereses: El manuscrito fue preparado y revisado con la participación de todos los autores, quienes declaramos que no existe ningún conflicto de intereses que ponga en riesgo la validez de los resultados presentados.

\section{REFERENCIAS}

1. BURITICÁ-CÉSPEDES, P.E. 1999. Directorio de patógenos y enfermedades de las plantas de importancia económica en Colombia. ICA-Universidad Nacional. 229p.

2. CAICEDO-GUERRERO, S.; BAQUERO-PENUUELA, J.E.; GUEVARA-AGUDELO, E.J.; LOPEZ-CARDONA, N.; TIBOCHA-ARDILA, Y.S.; REYES DIAZ, J.C. 2020. Agrosavia Primavera 11: nueva variedad de soya para suelos mejorados de la altillanura plana y el piedemonte llanero. Corporación Colombiana de Investigación Agropecuaria. Agrosavia. 29p.

https://doi.org/10.21930/agrosavia.brochure.7403961

3. CASTAÑO-ZAPATA, J. 2002. Principios básicos de fitoepidemiología. Editorial Universidad de Caldas (Manizales, Colombia). 398p.

4. DE LISI, V.; REZNIKOV, S.; GÓMEZ PONCE, E.N.; AGUAYSOL, N.; CLAPS, M.P.; BERNAL, M.; CHAVES,
S.; VILLAFAÑE, P.; MARTÍNEZ MONTEROS, M.J.; GONZÁLEZ, M.V.; PLOPER, L.D. 2016. Enfermedades en el cultivo de la soja y su manejo durante la campaña 2015/2016. EEAOC. p.116-124.

5. DIXON, L.J.; SCHLUB, R.L.; PERNEZNY, K.; DATNOFF, L.E. 2009. Host specialization and phylogenetic diversity of Corynespora cassiicola. Phytopathology. 99(9):1015-1027. https://doi.org/10.1094/PHYTO-99-9-1015

6. EDWARDS MOLINA, J.P.; PAUL, P.A.; AMORIM, L.; DA SILVA, L.H.C.P.; SIQUERI, F.V.; BORGES, E.P.; BALARDIN, R.S. 2019. Effect of target spot on soybean yield and factors affecting this relationship. Plant Pathology. 68(1):107-115.

https://doi.org/10.1111/ppa.12944

7. ESCOBAR, M.; REZNIKOV, S.; BLECKWEDEL, J.; DE LISI, V.; AGUAYSOL, C.; CLAPS, M.; CATALDO, D.; GONZÁLEZ, M.; GÓMEZ, C.; LÓPEZ, E.; MEJAIL, P.; GONZALEZ, V.; LEDESMA, F.; PLOPER, D. 2019. Evaluación del comportamiento de genotipos de soja frente a la mancha anillada (Corynespora cassiicola) en el noroeste argentino durante la campaña 2018-2019. El cultivo de la soja en el nordeste argentino. p.99-103.

8. GODOY, C.V.; UTIAMADA, C.M.; MEYER, M.C.; CAMPOS, H.D.; FORCELINI, C.A.; PIMENTA, C.B.; CASSETARI NETO, D.; JACCOUD FILHO, D.S.; BORGES, E.; DE ANDRADE JUNIOR, E.R.; SIQUERI, F.; JULIATTI, F.C.; FEKSA, H.R.; GRIGOLLI, J.F.J.; NUNES JUNIOR, J.; CARNEIRO, L.C.; SILVA, L.H.C.P. DA; SATO, L.N.; CANTERI, M.G.; MADALOSSO, M.; GOUSSAIN, M.; MARTINS, M.C.; BALARDIN, R.S.; FURLAN, S.H.; MONTECELLI, T.D.N.; CARLIN, V.J.; VENANCIO, W.S. 2015. Eficiencia de fungicidas para o controle da ferrugemasiática da soja, Phakopsora pachyrbizi, na safra 2014/15: resultados sumarizados dos ensaios cooperativos. Embrapa Soja. 1-6.

9. INSTITUTO COLOMBIANO AGROPECUARIO, ICA. 2013a. Resolución 004958 del 28 de noviembre de 2013 "Por la cual se ordena el Registro de la variedad de soja CORPOICA GUAYURIBA 9 de la Corporación Colombiana de Investigación Agropecuaria CORPOICA, en el Registro Nacional de Variedades Comerciales del ICA. $4 p$.

10. INSTITUTO COLOMBIANO AGROPECUARIO, ICA. 2013b. Resolución 004964 del 28 de noviembre de 2013 "Por la cual se ordena el Registro de la variedad de soja CORPOICA ACHAGUA 8 de la Corporación Colombiana de Investigación Agropecuaria CORPOICA, en el Registro Nacional de Variedades Comerciales del ICA. 4p.

11. KOENNING, S.R.; CRESWELL, T.C.; DUNPHY, E.J.; 
SIKORA, E.J.; MUELLER, J.D. 2006. Increased occurrence of target spot of soybean caused by Corynespora cassiicola in the southeastern United States. Plant Disease. 90(7):974.

https://doi.org/10.1094/PD-90-0974C

12. LÓPEZ-CARDONA, N.; GUEVARA, Y.A.; GAÑAN, L. 2020. First report of target spot of soybean caused by Corynespora cassiicola in the Colombian Eastern Plains. Plant Disease. 105(2):490.

https://doi.org/10.1094/PDIS-07-20-1586-PDN

13. PLOPER, L.D.; GONZALEZ, V.; DE LISI, V.; REZNIKOV, S.; HENRIQUEZ, D.D.; DEVANI, M.R. 2013. Research on target spot of soybean in northwestern Argentina. In: World Soybean Research Conference IX. (Durban, South África). Disponible desde Internet en:

https://www.proteinresearch.net/imgs/wsrc2013/20-

February-session-2/409_ploper-ld.pdf (con acceso 05/09/18).
14. SAKAMOTO, C.M.; SHAW, R.H. 1967. Light distribution in field soybean canopies. Agronomy Journal. 59(1):7-9. https://doi.org/10.2134/agronj1967.000219620059000100 $03 \mathrm{x}$

15. SINCLAIR, J.B. 1999. Target spot. In: Hartman, G.L. Rupe, J.C.; Sikora, E.J.; Domier, L.L.; Davis, J.A.; Steffey, K.L. (eds.). Compendium of soybean diseases. The American Phytopathological Society, St. Paul, MN. 27p.

16. SOARES, R.M.; GODOY, C.V.; OLIVEIRA, M.C.N. 2009. Escala diagramática para avaliação da severidade da mancha alvo da soja. Tropical Plant Pathology. 34(5):333-338. https:/ /dx.doi.org/10.1590/S1982-56762009000500007

17. WRATHER, J.A.; KOENNING, S.R. 2006. Estimates of disease effects on soybean yields in the United States 2003 to 2005. J. Nematology. 38(2):173-180. 